\title{
Improved Super-Orthogonal Trellis-Coded Spatial Modulation Using STBC-CSM
}

\author{
Ammar M. Abu-Hudrouss \\ Electrical Engineering Department, Islamic University of Gaza, Gaza, Palestine \\ Email: ahdrouss@iugaza.edu.ps
}

How to cite this paper: Abu-Hudrouss, A.M. (2018) Improved Super-Orthogonal Trellis-Coded Spatial Modulation Using STBC-CSM. Journal of Computer and Communications, 6, 149-156. https://doi.org/10.4236/jcc.2018.612015

Received: October 31, 2018

Accepted: December 24, 2018

Published: December 27, 2018

Copyright $\odot 2018$ by author and Scientific Research Publishing Inc. This work is licensed under the Creative Commons Attribution International License (CC BY 4.0).

http://creativecommons.org/licenses/by/4.0/

\section{cc) (i) Open Access}

\begin{abstract}
Trellis coded modulation (TCM) is a scheme that enhances the error performance without extra power not bandwidth. This paper presents a modified Super-Orthogonal Trellis-Coded Spatial Modulation (SOTC-SM) based on a cyclic structure of the Space Time Coding. The developed code benefits from expanded codebook of the Space Time Block Coded Spatial Modulation (STBC-SM) to enhance the coding gain. The set-partitioning and the code design based on the expanded codebook was given for codes with rate of 2 and 3 bps and can be easily extended to higher rates. The Bit-Error Rate (BER) performance of the proposed scheme was evaluated via computer simulation. It was shown that the proposed scheme outperforms the SOTC-SM performance for the same number of transmit antennas.
\end{abstract}

\section{Keywords}

Space Time Block Coding, Spatial Modulation, Trellis, Cyclic, STBC-SM, SOTC-SM, Set Partitioning

\section{Introduction}

There are increasing demands for high data rates in modern wireless communication systems. Therefore, researchers are trying their best to develop new technologies and protocols for maximizing both the throughput and spectral efficiency. These efforts are highly important to meet the requirements of the fifth generation wireless systems that are expected to be deployed beyond 2020. Multiple Input Multiple Output (MIMO) systems take advantage of transmitter multiple antennas to either send different data streams which provide multiplexing gain as the same data stream to achieve diversity gain [1]. However, multiplexing MIMO such as the Vertical Bell Laboratories Layered Space-Time (V-BLAST) [2] [3] scheme suffers from a substantial decoding complexity [4]. Space Time 
Block Codes are introduced by Alamouti in [5] to achieve transmit diversity without the knowledge of the channel stat information at the transmitter.

Space Shift Keying (SSK) [6] and Spatial Modulation(SM) [7] are novel developments that utilize the multiple antennas at the transmitter to provide multiplexing gain with lower complexity than the VBLAST [8]. The main advantages offered by the SSK and SM schemes are that no Inter-Antenna Synchronization (IAS) is required and the Inter-Channel Interference (ICI) does not form a problem at the receiver [8]. This is because only one single transmit antenna is activated in any symbol duration. However, both SSK and SM do not offer a transmit diversity.

Coherent Space-Time Shift Keying (CSTSK) in [9] introduces a flexible trade-off between the achievable diversity and multiplexing gain. Time-Orthogonal Signal Design assisted Spatial Modulation (TOSD-SM) [10] uses time-orthogonal shaping filters that attains a transmit diversity order of two. Space-Time Block Coded Spatial Modulation (STBC-SM) [11] [12] scheme uses an Alamouti STBC code for achieving a transmit diversity order of two.

Space Time Trellis Codes (STTCs) are introduced by Tarokh et al. [13] by joining the concept of multi-antenna system with channel coding. The STTCs introduce both diversity and coding gains compared to lower complexity space-time block codes which achieve only diversity gain. Instead of using rank and determinant criterion to determine the coding gain, Chen in [14] used the trace criterion which governs the coding for systems with large transmit and/or receive antennas. In [15], the authors introduced STTCs with better distance spectrum. Super orthogonal space trellis time codes (SO-STTC) has been introduced by Jafarkhani in [16]. The SO-STTCs outperform most of the previous STTCs and with lower complexity [17].

Spatial Modulation for trellis coding (SM-TC) has been introduced in [18]. In [19], the set partitioning of SO-STTCs is applied to space-time block coded Spatial Modulation to construct super orthogonal trellis coded spatial modulation (SOTC-SM). It has been shown in [19] that SOTC-SM achieves significantly better bit error rate (BER) and frame error rate (FER) performance than SM-TC. Quadrature spatial modulation has been introduced in [20] with higher spectral efficiency than conventional spatial modulation. Trellis codes based on QSM is introduced in [21] but it has no transmit diversity.

In this paper, instead of using the cyclic structure of STBC-CSM proposed in [22] to increase the data rate, it is used in this paper to expand the codebook of the trellis space time coded spatial modulation. This allows for superior performance with less number of transmit antenna compared to the code introduced in [19].

The rest of the paper is organized as follows. In Section 2, the STBC-CSM of [22] is explained. In Section 3, the modified trellis code structure based on STBC-CSM is introduced. In Section 4, the new code structure and different examples are explained. Finally, the results are presented in Section 5, and the 
paper is concluded in Section 6 .

\section{The Code Design}

In this section, the cyclic space time coded spatial modulation (STBC-CSM) which is the basic building block of our trellis code will be explained.

\subsection{STBC-CSM}

Alamouti in [4] has introduced a simple scheme to achieve transmitter space-time diversity without the channel state information being known at the transmitter. The scheme is developed for two transmit antennas where the transmission matrix is given by,

$$
X=\left[\begin{array}{cc}
s_{1} & s_{2} \\
-s_{2}^{*} & s_{1}^{*}
\end{array}\right]
$$

where $s_{1}$ and $s_{2}$ are the transmitted symbols drawn from PSK/QAM constellation. Space time block coded spatial modulation (STBC-SM) based on Alamouti matrix is introduced in [10]. In each time instance, two antennas are active and these antennas transmit the Alamouti matrix in two consecutive time slots. The indices of the active antennas are used for spatial multiplexing.

In, [18], the data rate of the STBC-SM is increased using cyclic structure of the transmitted matrix. The scheme is denoted as STBC-CSM. The STBC-CSM's transmission matrix for system with $N_{t}$ transmit antennas has the following structure,

$$
X_{i j}=\left[\begin{array}{rrrrrrrr}
0 & \cdots & s_{1} & 0 & \cdots & s_{2} 0 & \cdots & 0 \\
0 & \cdots & -s_{2}^{*} 0 & \cdots & s_{1}^{*} 0 & \cdots & 0
\end{array}\right],
$$

where the transmitting antenna indices for the Alamouti matrix are defined as $i$ and $j$ :

$$
\begin{gathered}
i=\left\{1,2, \cdots, N_{t}\right\}, \\
j=\left\{\begin{array}{ll}
i+k & \text { if } i+k \leq N_{t} \\
\bmod \left(i+k, N_{t}\right) & \text { if } i+k>N_{t}
\end{array},\right.
\end{gathered}
$$

For $k=1,2, \cdots, N_{t}$.

\subsection{SO-STBC and Set Partitioning}

The super orthogonal trellis codes introduced in [19] are based on Alamouticodes for two transmit antennas [4]. The transmission matrices are defined as,

$$
X=\left[\begin{array}{cc}
e^{j \theta} s_{1} & s_{2} \\
-e^{j \theta} s_{2}^{*} & s_{1}^{*}
\end{array}\right]
$$

where $\theta$ is chosen such as there is no expansion on the constellation size. If we consider the code with $\theta=0$ is the original code then the different values of $\theta$ are used to expand the code matrices. Instead of using the rotation angle, $\theta$, in [19], the expansion of the codebook is done in the spatial domain using a number of transmit antennas, $N_{t}>2$. The scheme is called super orthogonal trellis coded 
spatial modulation (SOTC-SM) where it is proven by computer simulation that the bit error rate of the SOTC-SM is significantly better than the SO-STBC for the same number of trellis states and transitions in expense of using higher number of transmit antennas.

The set partitioning for the trellis codes in the SOTC-SM is based on the coding gain difference (CGD).

If $c_{1}$ and $c_{2}$ are two different transmission-code matrices then the coding gaindistance (CGD) is defined as [14],

$$
\mathrm{CGD}=\operatorname{det}\left(B^{H}\left(c_{1} ; c_{2}\right) B\left(c_{1} ; c_{2}\right)\right),
$$

where $B\left(c_{1} ; c_{2}\right)$ is the difference between $c_{1}$ and $c_{2}$ and $(.)^{H}$ denotes the Hermitian complex conjugate transpose and "det" is the determinant. If the code is not full rank (not full diversity) the determinant can be replaced by the harmonic mean of eigenvalues of $B^{H} B$.

Example of set partitioning for the code with symbols drawn from the BPSK constellation can be found in Figure 1. The minimum CGD for $S, S_{a}$, and $S_{a b}$ are 4,4 , and 16; respectively. The set partitioning for STBC matrices with entry of QPSK and 8-PSK are listed in Table 1 and Table 2; respectively.

\subsection{Code Structure}

The code construction using the STBC-CSM is provided in this section. Code design for 2 and 3 bits $/ \mathrm{s} / \mathrm{Hz}$ is provided but codes with higher rates can be easily constructed using the same approach. The number of transmit antennas can also take any order but in the provided examples and for the sake of comparison with [19], 4 to 6 transmit antennas are used. An example of a trellis code is given in Figure 2 where each node in the figure represents a state, the element, $S_{i j}$ represents the transmission vector/matrix when we move from the state $i$ to the state $j$. As in [19], the new code structure is represented by $S \times S$ state transition matrices where $S$ is the number of Trellis states.

The entries of each transition matrix represent the parallel codewords that are transmitted from the $i^{\text {th }}$ state to the $j^{\text {th }}$ state, where $i$ is the row index and $j$ is the column index.

Example of 8 state code for $N_{t}=4$ is given in matrix 5. In Equation (6) and Equation (7) represents 8-state and 16-state code for $N_{t}=6$; respectively. The

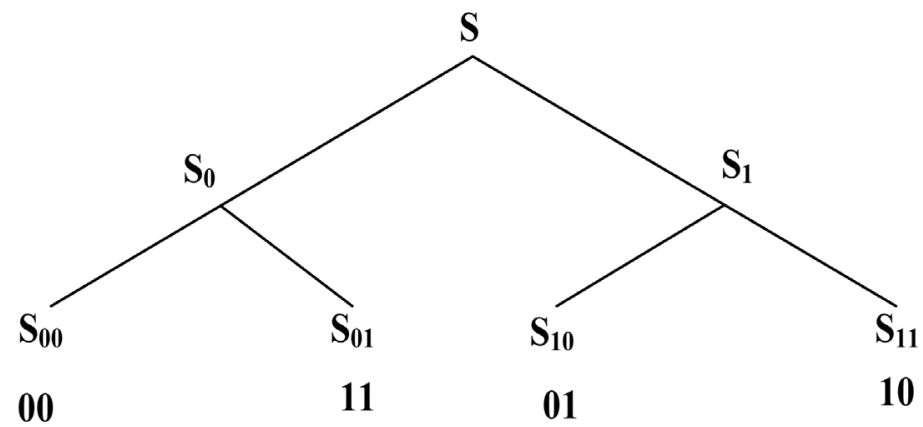

Figure 1. Set partitioning for BPSK Alamouti ST code. 
Table 1. Set partitioning for QPSK; the numbers represent the indices of the symbols in the STBC code.

\begin{tabular}{|c|c|c|c|c|c|c|c|}
\hline \multicolumn{8}{|c|}{$X$} \\
\hline \multicolumn{4}{|c|}{$X_{\mathrm{a}}$} & \multicolumn{4}{|c|}{$X_{\mathrm{b}}$} \\
\hline \multicolumn{2}{|c|}{$X_{\text {aа }}$} & \multicolumn{2}{|c|}{$X_{\mathrm{ab}}$} & \multicolumn{2}{|c|}{$X_{\mathrm{ba}}$} & \multicolumn{2}{|c|}{$X_{\mathrm{bb}}$} \\
\hline $\mathrm{X}_{\mathrm{aaa}}$ & $\mathrm{X}_{\mathrm{aab}}$ & $\mathrm{X}_{\mathrm{aba}}$ & $\mathrm{X}_{\mathrm{abb}}$ & $\mathrm{X}_{\text {baa }}$ & $\mathrm{X}_{\mathrm{bab}}$ & $\mathrm{X}_{\mathrm{bba}}$ & $\mathrm{X}_{\mathrm{bbb}}$ \\
\hline$(11,33)$ & $(13,31)$ & $(22,44)$ & $(24,42)$ & $(12,34)$ & $(14,32)$ & $(21,43)$ & $(23,41)$ \\
\hline
\end{tabular}

Table 2. Set partitioning for 8-PSK; the numbers represent the indices of the symbols in the STBC code.

\begin{tabular}{|c|c|c|c|c|c|c|c|}
\hline \multicolumn{8}{|c|}{$X$} \\
\hline \multicolumn{4}{|c|}{$X_{\mathrm{a}}$} & \multicolumn{4}{|c|}{$X_{\mathrm{b}}$} \\
\hline \multicolumn{2}{|c|}{$X_{\mathrm{aa}}$} & \multicolumn{2}{|c|}{$X_{\mathrm{ab}}$} & \multicolumn{2}{|c|}{$X_{\mathrm{ba}}$} & \multicolumn{2}{|c|}{$X_{\mathrm{bb}}$} \\
\hline$X_{\text {aаa }}$ & $X_{\text {aab }}$ & $X_{\mathrm{aba}}$ & $X_{\mathrm{abb}}$ & $X_{\text {baa }}$ & $X_{\text {bab }}$ & $X_{\text {bba }}$ & $X_{\mathrm{bbb}}$ \\
\hline$(11,33)$ & $(13,31)$ & $(22,44)$ & $(24,42)$ & $(12,34)$ & $(14,32)$ & $(21,43)$ & $(23,41)$ \\
\hline$(55,77)$ & $(57,75)$ & $(66,88)$ & $(68,86)$ & $(56,78)$ & $(58,76)$ & $(65,87)$ & $(67,85)$ \\
\hline$(15,37)$ & $(17,35)$ & $(26,48)$ & $(28,46)$ & $(16,38)$ & $(18,36)$ & $(25,47)$ & $(27,45)$ \\
\hline$(51,73)$ & $(53,75)$ & $(62,84)$ & $(62,84)$ & $(52,74)$ & $(54,72)$ & $(61,83)$ & $(63,81)$ \\
\hline
\end{tabular}

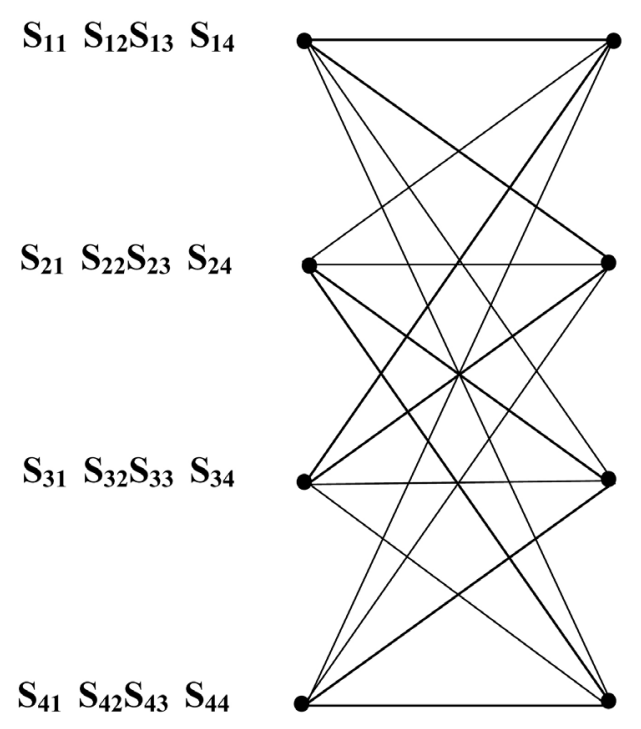

Figure 2. Trellis diagram for 4-state code.

entry, $X_{\mathrm{aba}}^{i j}$, is a matrix as in (2) where $i$ and $j$ represents the indices of nonzero columns and aba is a pointer to the transmitted symbols as in Table 1 and Table 2.

\section{Numerical Results and Discussion}

In this section, we present some simulation results for the SOTC-CSM system with different number of transmit antennas and make comparison with SOTC-SM. The Frame Error Rate (FER) performance of both schemes is evaluated through Monte Carlo Simulations for different spectral efficiencies, different number of transmit antennas, and/or different number of trellis states. The FER is depicted 
against the signal to noise ratio (SNR) of each receive antenna. The frame length is assumed to be 40 symbol. The comparison is done based on a fixed spectral efficiency. The FER of the SOTC-CSM with $N_{t}=4$ and $N_{t}=6$ is presented for $2 \mathrm{bps}$ in Figure 3. As seen from the figure, the SOTC-CSM provides SNR gain of 2.04 $\mathrm{dB}$ over the SOTC-CSM in case of $N_{t}=4$ at FER $=10^{-4}$ and a gain of $1.61 \mathrm{~dB}$ in case of $N_{t}=6$ for the same FER. In allthe former cases, the number of Trellis states is eight. For 16-state Trellis in (7) with $N_{t}=6$, the SOTC-CSM provide 1.29 $\mathrm{dB}$ gain at FER $=10^{-4}$ over the 8-state SOTC-CSM $\left(N_{t}=6\right)$ and a gain of $2.9 \mathrm{~dB}$ over 8-state SOTC-SM $\left(N_{t}=6\right)$.

The same is repeated for spectral efficiencies of 3 bps in Figure 4 with eight

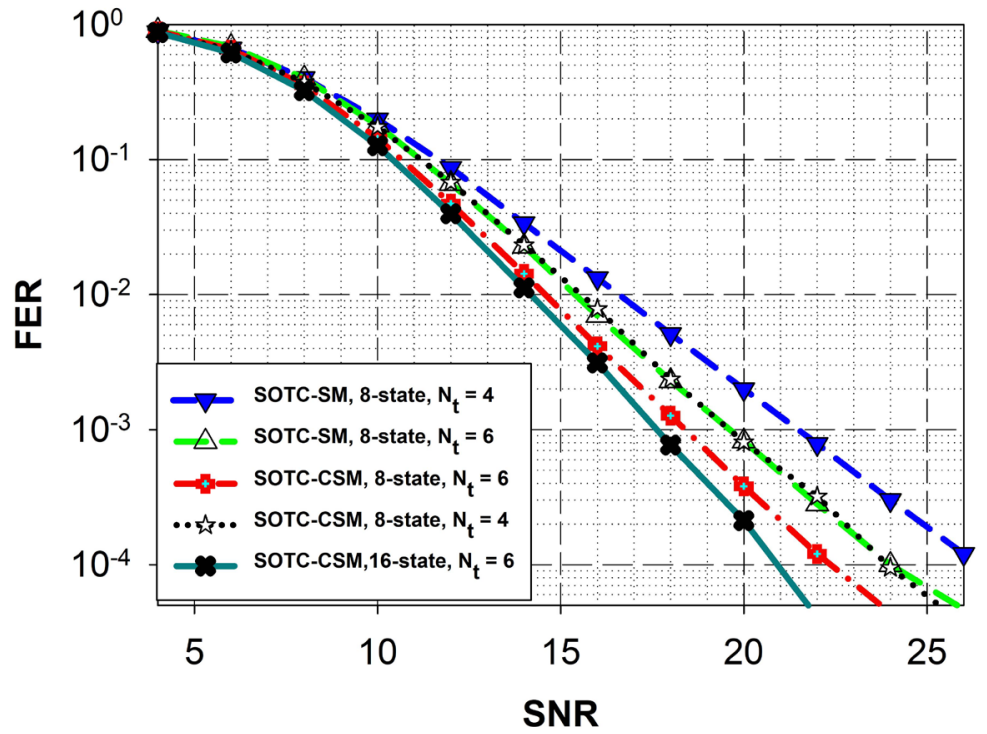

Figure 3. FER performance for 8-state and 16-state SOTC-CSM schemes at 2 bits/s/ $\mathrm{Hz}$ and $N_{t}$ of 4 and 6 .

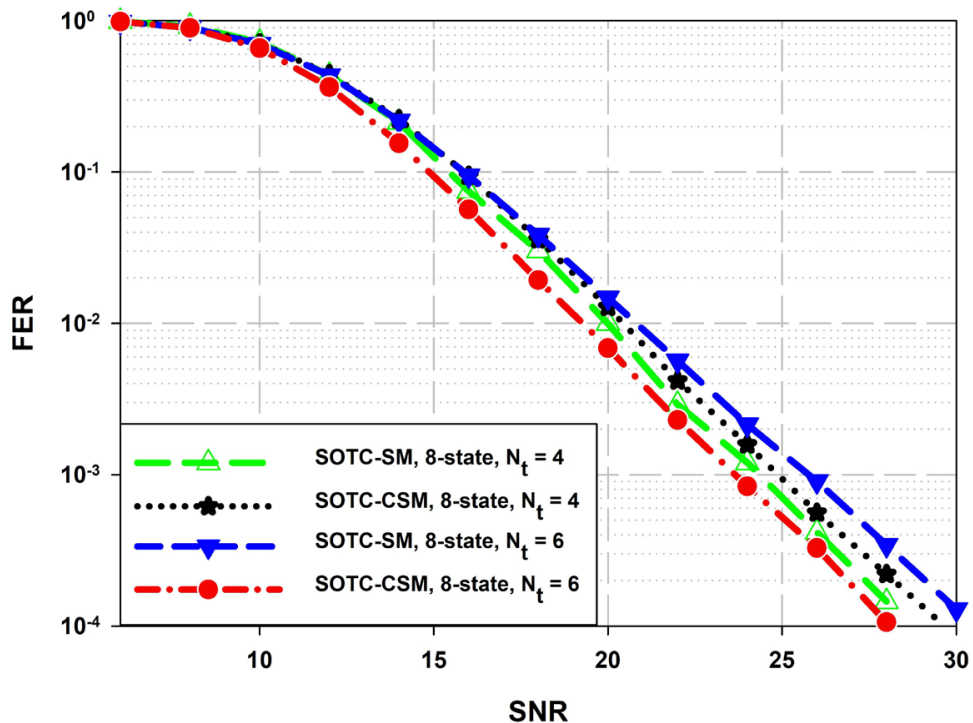

Figure 4. FER performance for 8 -state SOTC-CSM schemes at $3 \mathrm{bits} / \mathrm{s} / \mathrm{Hz}$ and $N_{t}$ of 4 and 6. 
Trellis states. As seen from the figure, the SOTC-CSM provides SNR gain of $1.02 \mathrm{~dB}$ over the SOTC-CSM in case of $N_{t}=4$ at FER $=2 \times 10^{-4}$ and a gain of 0.9 $\mathrm{dB}$ in case of $N_{t}=6$ for the same FER.

\section{Conclusion}

In this paper, we have introduced a modified version of SOTC-SM. The new code is denoted as SOTC-CSM is based on extended codebook provided by cyclic space time spatial codes. It has been shown through computer simulations that the proposed SOTC-CSM schemes achieve significantly better error performance than SOTC-CSM with the same decoding complexity. The research results allow for designating trellis codes based on better distributed distance spectrum STBC code.

\section{Conflicts of Interest}

The author declares no conflicts of interest regarding the publication of this paper.

\section{References}

[1] Mietzner, J., Schober, R., Lampe, L., Gerstacker, W.H. and Hoeher, P.A. (2009) Multiple-Antenna Techniques for Wireless Communications: A Comprehensive Literature Survey. IEEE Communications Surveys \& Tutorials, 11, 87-105. https://doi.org/10.1109/SURV.2009.090207

[2] Foschini, G.J. (1996) Layered Space-Time Architecture for Wireless Communication in a Fading Environment When Using Multiple Antennas. Bell Labs Technical Journal, 1, 41-59. https://doi.org/10.1002/bltj.2015

[3] Sugiura, S., Chen, S. and Hanzo, L. (2010) Coherent and Differential Space-Time Shift Keying: A Dispersion Matrix Approach. IEEE Transactions on Communications, 8, 3219-3230. https://doi.org/10.1109/TCOMM.2010.093010.090730

[4] Elshokry, A. and Abu-Hudrouss, A. (2012) Performance Evaluation of MIMO Spatial Multiplexing Detection Techniques. Al-Azhar Journal of Natural Science, 14, 47-60.

[5] Alamouti, S. (1998) A Simple Transmit Diversity Technique for Wireless Communications. IEEE Journal on Selected Areas in Communications, 16, 1451-1458. https://doi.org/10.1109/49.730453

[6] Jeganathan, J., Ghrayeb, A., Szczecinski, L. and Ceron, A. (2009) Space Shift Keying Moulation for MIMO Channels. IEEE Transactions on Wireless Communications, 8, 3692-3703. https://doi.org/10.1109/TWC.2009.080910

[7] Mesleh, R., Haas, H., Sinanovic, S., Ahn, C.W. and Yun, S. (2008) Spatial Modulation. IEEE Transactions on Vehicular Technology, 57, 2228-2241. https://doi.org/10.1109/TVT.2007.912136

[8] Rajashekar, S.R., Hari, K.V.S. and Hanzo, L. (2013) Structured Dispersion Matrices from Division Algebra Codes for Space-Time Shift Keying. IEEE Signal Processing Letters, 20, 371-374. https://doi.org/10.1109/LSP.2013.2247997

[9] Sugiura, S., Chen, S. and Hanzo, L. (2011) Generalized Space-Time Shift Keying Designed for Flexible Diversity-, Multiplexing- and Complexity-Tradeoffs. IEEE Transactions on Communications, 10, 1144-1153. 
[10] Di Renzo, M. and Haas, H. (2011) Space Shift Keying (SSK) Modulation: On the Transmit-Diversity/Multiplexing Trade-Off. IEEE International Conference on Communications (ICC), Kyoto, 5-9 June 2011, 1-6.

[11] Basar, E., Aygolu, U., Panayirci, E. and Poor, H.V. (2011) Space-Time Block Coded Spatial Modulation. IEEE Transactions on Communications, 59, 823-832. https://doi.org/10.1109/TCOMM.2011.121410.100149

[12] El Astal, M.-T., Al Habbash, A.H. and Abu-Hudrouss, A. (2018) Low-Complexity Detection for Space-Time Block Coded spatial Modulation Systems. 2018 International Conference on Promising Electronic Technologies (ICPET), Deir El-Balah, 3-4 October 2018, 49-51. https://doi.org/10.1109/ICPET.2018.00015

[13] Tarokh, V., Seshadri, N. and Calderbank, A. (1998) Space-Time Codes for High Data Rate Wireless Communication: Performance Criterion and Code Construction. IEEE Transactions on Information Theory, 44, 744-765.

https://doi.org/10.1109/18.661517

[14] Chen, Z., Yuan, J. and Vucetic, B. (2001) Improved Space-Time Trellis Coded Modulation Scheme on Slow Rayleigh Fading Channels. Electronics Letters, 37, 440-441. https://doi.org/10.1049/el:20010284

[15] Guixia, K., Ping, Z., Haas, H. and Schulz, E. (2002) Good Space-Time Trellis Codes in Terms of Distance Spectrum. Proceedings IEEE 56th Vehicular Technology Conference, Vancouver, 24-28 September 2002, 252-255.

[16] Jafarkhani, H. and Seshadri, N. (2003) Super-Orthogonal Space-Time Trellis Codes. IEEE Transactions on Information Theory, 49, 937-950.

https://doi.org/10.1109/TIT.2003.809607

[17] Yun, Z. and Jafarkhani, H. (2006) Super-Pseudo-Orthogonal Space-Time Trellis Codes. IEEE Wireless Communications and Networking Conference, Las Vegas, 3-6 April 2006, 1380-1385.

[18] Basar, E., Aygolu, U., Panayirci, E. and Poor, H.V. (2011) New Trellis Code Design for Spatial Modulation. IEEE Transactions on Wireless Communications, 10, 2670-2680. https://doi.org/10.1109/TWC.2011.061511.101745

[19] Basar, E., Aygolu, U., Panayirci, E. and Poor, H.V. (2012) Super-Orthogonal Trellis Spatial Modulation. IET Communications, 6, 2922-2932. https://doi.org/10.1049/iet-com.2012.0355

[20] Mesleh, R., Ikki, S. and Aggoune, H.M. (2015) Quadrature Spatial Modulation. IEEE Transactions on Vehicular Technology, 64, 2738-2742. https://doi.org/10.1109/TVT.2014.2344036

[21] Yigit, Z., Basar, E. and Mesleh, R. (2018) Trellis Coded Quadrature Spatial Modulation. Physical Communication, 29, 147-155. https://doi.org/10.1016/j.phycom.2018.05.007

[22] Li, X.F. and Wang, L. (2014) High Rate Space-Time Block Coded Spatial Modulation with Cyclic Structure. IEEE Communications Letters, 18, 532-535. https://doi.org/10.1109/LCOMM.2014.012714.132544 